gen wird. In ein bis zwei Jahren werden wir Genaueres wissen, welcher miRNA-Cocktail diagnostisch am treffsichersten ist und ob Vorteile gegenüber dem Troponin zu erwarten sind. Der große Vorteil der miRNAs ist ihre Spezifität: Es gibt Varianten, die nur in der Herzmuskelzelle vorkommen, andere, die nur in Fibroblasten oder Endothelzellen stark exprimiert sind. Je nachdem, welche miRNA-Typen im Blut zu finden sind, zeigt sich, ob vielleicht eine Fibrose im Vordergrund steht, ob die Kapillarisierung unzureichend ist oder auch wie viele Muskelzellen beim Infarkt abgestorben sind - alle diese Befunde liefern uns genauere pathophysiologische und auch funktionelle Informationen als das Troponin.

\section{Modifikation durch Medikamente CardioVasc: Können die miRNAs durch Me- dikamente beeinflusst werden?}

Thum: Dazu gibt es erst präliminäre Befunde.
Die Expression von miRNAs kann durch ACEHemmer oder Statine beeinflusst werden, sodass ich davon ausgehe, dass die günstigen kardialen Effekte von Pharmaka zum Teil auch über miRNAs vermittelt werden.

Genauer untersucht haben wir Nitrate, allerdings in vitro an kultivierten Endothelzellen.

Diese Zellen können Stressfaktoren ausgesetzt werden, dann werden Stress-miRNAs hochreguliert. Diesen Vorgang kann man nun nutzen, um die Einwirkung bestimmter Pharmaka, in unserem Fall von Langzeitnitraten, zu studieren. Wir haben festgestellt, dass sich PETN in diesem Szenario als günstig erwies im Vergleich zu den Vergleichsubstanzen. PETN hat die Stressreaktion attenuiert, abgeschwächt.

Hoffnung auf bessere Diagnostika und Therapeutika auf genetischer Basis CardioVasc: Mit den miRNAs befassen sich nicht nur die Kardiologen, dieses neue For- schungsfeld durchzieht inzwischen fast die gesamte Medizin. Welche Perspektiven sind heute schon realistisch?

Thum: Zum einen hofft man, wie diskutiert, auf verbesserte diagnostische Tools. Zum anderen wird versucht, die miRNAs als therapeutische Targets zu nutzen, wir arbeiten auch daran. Tatsächlich gibt es weltweit schon klinische Studien in Phase 1 und Phase 2, die miRNA-Antagonisten verwenden, genetische Medikamente, die miRNAs im Körper beeinflussen können. Solche Studien laufen z. B. in der Hepatologie zur Therapie der Hepatitis C. Wir haben uns in Hannover auf kardiologische Fragestellungen konzentriert. Wir wollen miRNA-Therapeutika entwickeln mit dem Ziel, das miRNA-Muster nach Infarkten wieder zu normalisieren und dadurch auch Herz und Kreislauf.

Die Fragen stellte Jochen Aumiller

MikroRNA und Herzinfarkt

\title{
Nummer 24 orchestriert ein ganzes Gennetzwerk
}

Forscher der MHH haben herausgefunden, dass MikroRNA-24 (MiR-24) im Herzen auf ein ganzes Gennetzwerk wirkt und so die Auswirkungen des Myokardinfarkts verstärkt. In Zell- und Tierversuchen konnte nachgewiesen werden, dass das Ausschalten von MiR-24 einen positiven Effekt hat. Die Arbeit von Thomas Thum und Jan Fiedler wurde in Circulation veröffentlicht.

Die Forschergruppe fand heraus, das MiR-24 eine wichtige Rolle nach einem Myokardinfarkt im Infarktgewebe übernimmt. Es ist dort aktiviert und reguliert eine Reihe von Genen. Die Forscher wiesen in Versuchen mit Zebrafischen nach, welche Gene von MiR-24 gesteuert werden. „Wir konnten zeigen, dass MiR-24 einen schädigenden Einfluss hat", so Thum , denn als Folge dieser Veränderungen in der Genregulation gehen in der Infarktregion Endothelzellen des Herzens zu Grunde. Zudem wird die Kapillarbildung unterdrückt." Der Zelltod von Gefäßzellen führt zu einer größeren Narbenbildung am Herzen. Werden kaum Kapillaren gebildet, ist die Durchblutung noch stärker unterbunden.
Antagonist von MiR24 im Tierversuch Will man die schädigende Wirkung bekämpfen, muss MiR-24 ausgeschaltet werden. Die Forscher haben in Kooperation mit einer Pharmafirma den Wirkstoff AntagomiR-24 getestet. Dabei handelt es sich um eine Negativform von MiR-24, das sich in einem SchlüsselSchloss-Prinzip an die Mikro-RNA anlagert und sie dadurch blockiert. „Bei Mäusen, die einen Herzinfarkt erlitten hatten, konnten wir zeigen, dass sich nach einer zweimaligen Therapie die Kapillarbildung deutlich verbessert hat, also mehr Gefäße vorhanden waren", erklärt Thum. Zudem sei die Herzfunktion deutlich besser geworden: Im Vergleich zur Kontrollgruppe war in der behandelten Gruppe die Anzahl der Gefäße doppelt so hoch und der schädigende Effekt der Herzinfarktes auf die Pumpfunktion um 50\% gemindert. Die Gefahr, zusätzlich ein Lungenödem zu erleiden, also Wassereinlagerungen in die Lunge als Folge eines Herzinfarkts, konnte dank der Therapie um 40\% gesenkt werden.
Gegen Herzinfarkt und anderes

Die Forscher konnten mit ihrer Arbeit also nicht nur neue Mechanismen der Endothelapoptose und Vaskularisierung im Herzen aufklären, sondern sie liefern zudem einen Ansatz für eine neue Herzinfarkttherapie. „Das Medikament könnte direkt während einer Katheteruntersuchung oder Bypassoperation in das betroffene Gewebe gespritzt werden", sagt Thum. Bevor die Therapie aber beim Menschen zum Einsatz kommen kann, müssen in weiteren Versuchen die Langzeitwirkung und mögliche Toxizitäten geklärt werden. Die Forscher rechnen mit einer klinischen Anwendung frühestens in fünf Jahren. Die muss dann aber nicht auf den Herzinfarkt beschränkt bleiben. „MiR-24 wirkt sicher auch in weiteren Organen", betont Thum, , jetzt gehen wir auch auf die Suche nach anderen Einsatzmöglichkeiten."

II Quelle: Medizinischen Hochschule Hannover. Fiedler J, et al. Circulation. 2011;124(6):720-30; doi:10.1161/ CIRCULATIONAHA.111.039008. 